\title{
QUANTUM PERMUTATION GROUPS: A SURVEY
}

\author{
TEODOR BANICA \\ Department of Mathematics, Paul Sabatier University \\ 118 route de Narbonne, 31062 Toulouse, France \\ E-mail: banica@picard.ups-tlse.fr \\ JULIEN BICHON \\ Department of Mathematics, Blaise Pascal University \\ Campus des Cezeaux, 63177 Aubière Cedex, France \\ E-mail: Julien.Bichon@math.univ-bpclermont.fr \\ BENOÎT COLLINS \\ Department of Mathematics, Claude Bernard University \\ 43 bd du 11 novembre 1918, 69622 Villeurbanne, France \\ E-mail:collins@math.univ-lyon1.fr
}

\begin{abstract}
This is a presentation of recent work on quantum permutation groups. Contains: a short introduction to operator algebras and Hopf algebras; quantum permutation groups, and their basic properties; diagrams, integration formulae, asymptotic laws, matrix models; the hyperoctahedral quantum group, free wreath products, quantum automorphism groups of finite graphs, graphs having no quantum symmetry; complex Hadamard matrices, cocycle twists of the symmetric group, quantum groups acting on 4 points; remarks and comments.
\end{abstract}

1. Introduction. The idea of noncommuting coordinates goes back to Heisenberg, who was in turn motivated by results of Balmer and Ritz-Rydberg regarding spectra of chemical elements. Several theories emerged from Heisenberg's work, most complete being Connes' noncommutative geometry, where the base space is a Riemannian manifold. See [30].

The specific idea of using algebras of free coordinates on algebraic groups should be attributed to Brown [25]. The point is the following: given a group $G \subset U_{n}$, the matrix coordinates $u_{i j} \in C(G)$ commute with each other, and satisfy certain relations $R$. One

2000 Mathematics Subject Classification: Primary 46L65; Secondary 46L37, 46L54, 46L87.

Key words and phrases: quantum permutation group, magic unitary matrix.

The paper is in final form and no version of it will be published elsewhere. 
can define then the universal algebra generated by abstract variables $u_{i j}$, subject to the relations $R$. The spectrum of this algebra is an abstract object, called noncommutative version of $G$. The noncommutative version is not unique, because it depends on $R$.

A detailed study of Brown's algebras, from a K-theoretic point of view, is due to McClanahan [42]. Unfortunately, the whole subject is a bit limited, because Brown's choice for the relations $R$ is somehow minimal, and this makes the corresponding algebra too big. This algebra has a comultiplication and a counit, but no antipode. In other words, the corresponding noncommutative version is a quantum semigroup.

The continuation of story makes use of Woronowicz's axiomatization of compact quantum groups [58], [59]. The algebras $A_{o}(n)$ and $A_{u}(n)$, corresponding to the orthogonal and unitary groups, appeared in Wang's thesis [55]. Then Connes suggested use of symmetric groups, and the algebra $A_{s}(n)$ was constructed in [56]. In all three cases the idea is the same as Brown's. The point is to carefully choose the relations $R$, in order to get a compact quantum group in the sense of Woronowicz.

The spectrum of $A_{s}(n)$ is called free version of $S_{n}$. This a compact quantum group, bigger than $S_{n}$. Its subgroups are called quantum permutation groups.

In this paper we present a number of known facts about such quantum groups. We focus on combinatorial aspects, and their algebraic or probabilistic interpretation.

Acknowledgements. This work was started at Będlewo in October 2006 at the workshop "Noncommutative harmonic analysis with applications to probability". We would like to thank Marek Bożejko for the invitation, and for several stimulating discussions.

2. Operator algebras. The operator algebra background needed in order to construct quantum permutation groups reduces to the definition of $C^{*}$-algebras, and to some early work on the subject. We thought it useful to include a short presentation of this material. Actually the present text is written as to be at the same time an introduction and survey paper.

Definition 2.1. A $C^{*}$-algebra is a complex algebra with unit, having a norm and an involution, such that Cauchy sequences converge, and such that $\left\|a a^{*}\right\|=\|a\|^{2}$.

The basic example is $B(H)$, the algebra of bounded operators on a Hilbert space $H$. The GNS theorem states that any $C^{*}$-algebra appears as subalgebra of some $B(H)$.

The key example is $C(X)$, the algebra of continuous functions on a compact space $X$. The Gelfand theorem below states that any commutative $C^{*}$-algebra is of this form.

We need some basic spectral theory. The spectrum of an element $a \in A$ is the set $\sigma(a)$ consisting of complex numbers $\lambda$ such that $a-\lambda$ is not invertible. The spectral radius $\rho(a)$ is the radius of the smallest disk centered at 0 containing $\sigma(a)$.

TheOREm 2.1. Let $A$ be a $C^{*}$-algebra.

1. The spectrum of a norm one element is in the unit disk.

2. The spectrum of a unitary element $\left(a^{*}=a^{-1}\right)$ is on the unit circle.

3. The spectrum of a self-adjoint element $\left(a=a^{*}\right)$ consists of real numbers.

4. The spectral radius of a normal element $\left(a a^{*}=a^{*} a\right)$ is equal to its norm. 
The first assertion follows from the formula $1 /(1-x)=1+x+x^{2}+\ldots$

If $f$ is a rational function having poles outside $\sigma(a)$, we have $\sigma(f(a))=f(\sigma(a))$. By using the functions $z^{-1}$ and $(z+i t) /(z-i t)$ we get the middle assertions.

Finally, the inequality $\rho(a) \leq\|a\|$ is clear from the first assertion. For the converse we fix $\rho>\rho(a)$, and we integrate over the circle of radius $\rho$ :

$$
\int \frac{z^{n}}{z-a} d z=\sum_{k=0}^{\infty}\left(\int z^{n-k-1} d z\right) a^{k}=a^{n} .
$$

By applying the norm and taking $n$-th roots we get $\rho \geq \lim \left\|a^{n}\right\|^{1 / n}$.

In the case $a=a^{*}$ we have $\left\|a^{n}\right\|=\|a\|^{n}$ for any exponent of the form $n=2^{k}$, and by taking $n$-th roots we get $\rho \geq\|a\|$. This gives the missing inequality $\rho(a) \geq\|a\|$.

In the general case $a a^{*}=a^{*} a$ we have $a^{n}\left(a^{n}\right)^{*}=\left(a a^{*}\right)^{n}$, and we get $\rho(a)^{2}=\rho\left(a a^{*}\right)$. Now since $a a^{*}$ is self-adjoint, we get $\rho\left(a a^{*}\right)=\|a\|^{2}$, and we are done.

ThEOREM 2.2. The commutative $C^{*}$-algebras are those of form $C(X)$.

The proof is as follows. Given a commutative $C^{*}$-algebra $A$, we can define $X$ to be the set of characters $\chi: A \rightarrow \mathbb{C}$, with topology making continuous all evaluation maps $e_{a}$. Then $X$ is a compact space, and $a \mapsto e_{a}$ is a morphism of algebras $e: A \rightarrow C(X)$. We prove first that $e$ is involutive. We use the following formula:

$$
a=\frac{a+a^{*}}{2}-i \cdot \frac{i\left(a-a^{*}\right)}{2} .
$$

Thus it is enough to prove the equality $e_{a^{*}}=e_{a}^{*}$ for self-adjoint elements $a$. But this is the same as proving that $a=a^{*}$ implies that $e_{a}$ is a real function, which is in turn true, because $e_{a}(\chi)=\chi(a)$ is an element of $\sigma(a)$, contained in the reals.

Since $A$ is commutative, each element is normal, so $e$ is isometric: $\left\|e_{a}\right\|=\rho(a)=\|a\|$.

It remains to prove that $e$ is surjective. But this follows from the Stone-Weierstrass theorem, because $e(A)$ is a closed subalgebra of $C(X)$, which separates points.

3. Hopf algebras. This is a short introduction to Hopf algebra philosophy.

In order to simplify presentation, we call comultiplication, counit and antipode any morphisms of $C^{*}$-algebras of the following type:

$$
\begin{aligned}
\Delta: A & \rightarrow A \otimes A, \\
\varepsilon: A & \rightarrow \mathbb{C}, \\
S: A & \rightarrow A^{o p} .
\end{aligned}
$$

The terminology comes from the fact that in the commutative case $A=C(X)$, the morphism $\Delta$ is transpose to a binary operation, or multiplication, $X \times X \rightarrow X$.

Definition 3.1. A finite Hopf algebra is a finite dimensional $C^{*}$-algebra, endowed with a comultiplication, counit and antipode, satisfying the following conditions:

$$
\begin{aligned}
(\Delta \otimes i d) \Delta & =(i d \otimes \Delta) \Delta, \\
(\varepsilon \otimes i d) \Delta & =i d, \\
(i d \otimes \varepsilon) \Delta & =i d, \\
m(S \otimes i d) \Delta & =\varepsilon(.) 1,
\end{aligned}
$$




$$
m(i d \otimes S) \Delta=\varepsilon(.) 1 .
$$

The group algebra $C^{*}(G)$ is the complex vector space spanned by $G$, with product $g \cdot h=g h$, involution $g^{*}=g^{-1}$, and norm coming from the regular representation.

We say that $A$ is cocommutative if $\Sigma \Delta=\Delta$, where $\Sigma(a \otimes b)=b \otimes a$.

THEOREM 3.1. Let $G$ be a finite group.

1. $C(G)$ is a commutative finite Hopf algebra, with

$$
\begin{aligned}
\Delta(\varphi) & =(g, h) \mapsto \varphi(g h), \\
\varepsilon(\varphi) & =\varphi(1), \\
S(\varphi) & =g \mapsto \varphi\left(g^{-1}\right)
\end{aligned}
$$

as structural maps. Any commutative finite Hopf algebra is of this form.

2. $C^{*}(G)$ is a cocommutative finite Hopf algebra, with

$$
\begin{aligned}
\Delta(g) & =g \otimes g, \\
\varepsilon(g) & =1, \\
S(g) & =g^{-1}
\end{aligned}
$$

as structural maps. Any cocommutative finite Hopf algebra is of this form.

In this statement the fact that $\Delta, \varepsilon, S$ satisfy the axioms is clear from definitions.

The assertion about commutative Hopf algebras follows from the Gelfand theorem. For the remaining assertion, let $A$ be a finite Hopf algebra, and consider its comultiplication, counit, multiplication, unit and antipode. By taking duals, we get linear maps as follows:

$$
\begin{aligned}
\Delta^{*} & : A^{*} \otimes A^{*} \rightarrow A^{*}, \\
\varepsilon^{*} & : \mathbb{C} \rightarrow A^{*}, \\
m^{*} & : A^{*} \rightarrow A^{*} \otimes A^{*}, \\
u^{*} & : A^{*} \rightarrow \mathbb{C}, \\
S^{*} & : A^{*} \rightarrow A^{*} .
\end{aligned}
$$

It is routine to check that these maps make $A^{*}$ into a finite Hopf algebra. Moreover, if $A$ is cocommutative then $A^{*}$ is commutative, so we can apply the first result. We get $A^{*}=C(G)$ for a certain finite group $G$, which in turn gives $A=C^{*}(G)$.

4. Compact quantum groups. There are several types of compact quantum groups. The formalism that we need is that of compact quantum groups whose square of the antipode is the identity. A first study here is due to Enock and Schwartz [37]. We use in this paper an adaptation of Woronowicz's axioms in [58].

Definition 4.1. A finitely generated Hopf algebra is a $C^{*}$-algebra $A$, together with a unitary matrix $u \in M_{n}(A)$ whose coefficients generate $A$, such that the formulae

$$
\begin{aligned}
\Delta\left(u_{i j}\right) & =\sum u_{i k} \otimes u_{k j}, \\
\varepsilon\left(u_{i j}\right) & =\delta_{i j}, \\
S\left(u_{i j}\right) & =u_{j i}^{*}
\end{aligned}
$$

define a comultiplication, a counit and an antipode. 
The maps $\Delta$ and $\varepsilon$ satisfy the usual axioms for a comultiplication and a counit. The map $S$ satisfies the usual axioms for an antipode, on the dense *-algebra generated by entries of $u$. Observe that the square of the antipode is the identity: $S^{2}=i d$.

Once the pair $(A, u)$ is given, the maps $\Delta, \varepsilon, S$ can exist or not. If they exist, they are uniquely determined, and we have a Hopf algebra. This point of view, somehow opposite to the spirit of abstract group theory, was invented by Woronowicz [58].

The terminology and axioms are motivated by the following result.

THEOREM 4.1. The following are finitely generated Hopf algebras.

1. $C(G)$, with $G \subset U_{n}$ compact Lie group.

2. $C^{*}(G)$, with $F_{n} \rightarrow G$ finitely generated group.

In both cases, we have to exhibit a certain matrix $u$. For the first assertion, we can use the matrix $u=\left(u_{i j}\right)$ formed by matrix coordinates of $G$, given by:

$$
g=\left(\begin{array}{lll}
u_{11}(g) & & u_{1 n}(g) \\
& \ddots & \\
u_{n 1}(g) & & u_{n n}(g)
\end{array}\right) .
$$

The second assertion is clear by using the diagonal matrix formed by generators:



The algebras in the above statement can be characterized as being the commutative or cocommutative finitely generated Hopf algebras. See Woronowicz [58].

In the general case we have the following heuristic formulae.

1. $A=C(G)$, with $G$ compact quantum group.

2. $A=C^{*}\left(G^{\prime}\right)$, with $G^{\prime}$ discrete quantum group.

Needless to say, the quantum groups $G, G^{\prime}$ don't exist as concrete objects. This is in fact the case with all kinds of quantum groups. See Drinfeld [35].

5. Free quantum groups. We construct now the orthogonal, unitary and symmetric quantum groups, following Wang's papers [55], [56]. Let $u \in M_{n}(A)$ be a square matrix over a $C^{*}$-algebra.

1. $u$ is called orthogonal if $u=\bar{u}$ and $u^{t}=u^{-1}$.

2. $u$ is called biunitary if $u^{*}=u^{-1}$ and $u^{t}=\bar{u}^{-1}$.

For the algebras $C\left(O_{n}\right)$ and $C\left(U_{n}\right)$, the corresponding matrix $u$ is orthogonal, respectively biunitary. In the symmetric group case the situation is less obvious. When using the embedding $S_{n} \subset U_{n}$ given by permutation matrices, the functions $u_{i j}$ are:

$$
u_{i j}=\chi\left\{\sigma \in S_{n} \mid \sigma(j)=i\right\} .
$$

These characteristic functions satisfy a condition which reminds magic squares. 
Definition 5.1. $u \in M_{n}(A)$ is called magic unitary if all entries $u_{i j}$ are projections, and on each row and column of $u$ these projections are orthogonal, and sum up to 1.

With these definitions in hand, it is routine to check that we have the following equalities, where $C_{\text {com }}^{*}$ means universal commutative $C^{*}$-algebra:

$$
\begin{aligned}
& C\left(O_{n}\right)=C_{\text {com }}^{*}\left(u_{i j} \mid u=n \times n \text { orthogonal }\right), \\
& C\left(U_{n}\right)=C_{\text {com }}^{*}\left(u_{i j} \mid u=n \times n \text { biunitary }\right), \\
& C\left(S_{n}\right)=C_{c o m}^{*}\left(u_{i j} \mid u=n \times n \text { magic unitary }\right) .
\end{aligned}
$$

In other words, orthogonality, biunitarity and magic unitarity are the relevant conditions about matrix coordinates of $O_{n}, U_{n}, S_{n}$. We can proceed now with liberation.

THEOREM 5.1. The universal algebras

$$
\begin{aligned}
& A_{o}(n)=C^{*}\left(u_{i j} \mid u=n \times n \text { orthogonal }\right), \\
& A_{u}(n)=C^{*}\left(u_{i j} \mid u=n \times n \text { biunitary }\right), \\
& A_{s}(n)=C^{*}\left(u_{i j} \mid u=n \times n \text { magic unitary }\right)
\end{aligned}
$$

are finitely generated Hopf algebras.

The proof is as follows. Let us use the generic term "special" for the three unitarity notions in the above theorem. Consider now the following three matrices, having coefficients in the target algebras of the maps $\Delta, \varepsilon, S$ to be constructed:

$$
\begin{aligned}
(\Delta u)_{i j} & =\sum u_{i k} \otimes u_{k j}, \\
(\varepsilon u)_{i j} & =\delta_{i j}, \\
(S u)_{i j} & =u_{j i}^{*} .
\end{aligned}
$$

The matrix $\varepsilon u=1$ is special, and it is routine to check that $\Delta u$ and $S u$ are special as well. Thus the maps $\varphi=\Delta, \varepsilon, S$ can be defined by $\varphi\left(u_{i j}\right)=(\varphi u)_{i j}$.

Summarizing, we have now free analogues of $O_{n}, U_{n}, S_{n}$. Their construction might seem quite mysterious, and indeed so it is: free quantum groups are not axiomatized.

The orthogonal and unitary algebras have the following properties.

1. $A_{o}(2)$ corresponds to the quantum group $S U_{2}^{-1}$.

2. $A_{o}(n)$ corresponds to an $R$-matrix quantization of $S U_{2}$.

3. $A_{u}(n)$ embeds into the free product $C(T) * A_{o}(n)$.

We refer to [10], [49] for an updated discussion of these results.

6. Quantum permutation groups. The algebra $A_{s}(n)$ is a free analogue of $C\left(S_{n}\right)$. We show now that the corresponding compact quantum group consists indeed of "quantum permutations".

The permutations of $S_{n}$ act on points of $X=\{1, \ldots, n\}$. The corresponding action map $(i, \sigma) \mapsto \sigma(i)$ gives by transposition a certain morphism $\alpha_{\text {com }}$, called coaction. This coaction can be expressed in terms of the magic unitary associated to $C\left(S_{n}\right)$ :

$$
\alpha_{c o m}\left(\delta_{i}\right)=\sum \delta_{j} \otimes u_{j i} .
$$

Now let $u$ be the magic unitary associated to $A_{s}(n)$, and consider the linear map $\alpha$ given by the above formula. Then $\alpha$ is a coaction, and we have the following result. 
THEOREM 6.1. We have the following commutative diagram:



Moreover, $\alpha$ is the universal Hopf algebra coaction on $X$.

This result appeared in Wang's paper [56], in a slightly different form. We refer to [2] for a detailed discussion, by using the magic unitarity condition.

At this point of writing, it is not clear whether quantum permutations do exist. The question is whether the canonical map $A_{s}(n) \rightarrow C\left(S_{n}\right)$ is an isomorphism or not.

THEOREM 6.2. Quantum permutations exist starting from $n=4$. More precisely:

1. For $n=1,2,3$ we have $A_{s}(n)=C\left(S_{n}\right)$.

2. For $n \geq 4$ the algebra $A_{s}(n)$ is not commutative, and infinite dimensional.

The first assertion follows from the fact that for $n=1,2,3$, the entries of a $n \times n$ magic unitary matrix have to commute with each other. This is clear for $n=1$, and also for $n=2$, where the magic unitary must be of the following special form:

$$
u_{p}=\left(\begin{array}{cc}
p & 1-p \\
1-p & p
\end{array}\right)
$$

At $n=3$ the proof is quite tricky. The idea is to use the Fourier transform over $Z_{3}$. In terms of the vector $\xi$ formed by third roots of unity, we can write $\alpha$ as follows:

$$
\alpha\left(\xi^{i}\right)=\sum \xi^{j} \otimes v_{j i}
$$

Now the magic unitarity condition on $u$ translates into a certain condition on $v$, and the point is that with this new condition, commutativity is clear. See [2].

At $n=4$ we use the following matrix, where $p, q$ are projections:

$$
u_{p q}=\left(\begin{array}{cccc}
p & 1-p & 0 & 0 \\
1-p & p & 0 & 0 \\
0 & 0 & q & 1-q \\
0 & 0 & 1-q & q
\end{array}\right)
$$

This shows that the algebra $\langle p, q\rangle$, which can be chosen to be not commutative and infinite dimensional, is a quotient of $A_{s}(4)$. This gives the last assertion.

The reasons why we have $A_{s}(4) \neq C\left(S_{4}\right)$ might remain quite mysterious. In what follows we propose several explanations for this fact.

7. The Temperley-Lieb algebra. We present here a first conceptual explanation for the main result in previous section. The idea is that $n=4$ is the critical value of the Jones index [40].

Consider the algebra $A_{s}(n)$. Its generators $u_{i j}$ are coefficients of the coaction $\alpha$, so the matrix $u$ is a corepresentation. The tensor powers of $u$ are defined as follows:

$$
\left(u^{\otimes k}\right)_{i_{1} \ldots i_{k}, j_{1} \ldots j_{k}}=u_{i_{1} j_{1}} \ldots u_{i_{k} j_{k}} .
$$


The problem is to compute the Hom spaces between these corepresentations.

Definition 7.1. The Temperley-Lieb algebra is given by

$$
T L_{n}(k, l)=\operatorname{span}\left\{\begin{aligned}
\cdots & \leftarrow 2 k \text { points } \\
W & \leftarrow k+l \text { strings } \\
\cdots & \leftarrow 2 l \text { points }
\end{aligned}\right\}
$$

where strings join pairs of points, do not cross, and are taken up to isotopy.

$T L_{n}$ is a tensor category: the composition is by vertical concatenation, with the rule that closed circles are deleted and replaced by the number $n$, the tensor product is by horizontal concatenation, and the involution is by upside-down turning of diagrams.

Temperley-Lieb diagrams act on tensors according to the following formula, where the middle symbol is 1 if all strings of $p$ join pairs of equal indices, and is 0 if not:

$$
p\left(e_{i_{1}} \otimes \ldots \otimes e_{i_{k}}\right)=\sum_{j_{1} \ldots j_{l}=1}^{n}\left(\begin{array}{c}
i_{1} i_{1} \ldots i_{k} i_{k} \\
p \\
j_{1} j_{1} \ldots j_{l} j_{l}
\end{array}\right) e_{j_{1}} \otimes \ldots \otimes e_{j_{l}} .
$$

In case the index satisfies $n \geq 4$, different diagrams produce different linear maps, and this action makes $T L_{n}$ a subcategory of the category of Hilbert spaces.

THEOREM 7.1. We have the equality of tensor categories

$$
\operatorname{Hom}\left(u^{\otimes k}, u^{\otimes l}\right)=T L_{n}(k, l)
$$

where $u$ is the fundamental corepresentation of $A_{s}(n)$, with $n \geq 4$.

The proof uses Woronowicz's duality in [59]. The idea is that the definition of $A_{s}(n)$ translates into a presentation result for the corresponding tensor category.

1. The fact that a unitary matrix $u$ is magic is equivalent to $M \in \operatorname{Hom}\left(u^{\otimes 2}, u\right)$ and $U \in \operatorname{Hom}(1, u)$, where $M, U$ are the multiplication and unit of $C^{n}$.

2. The relations satisfied by $M, U$ in a categorical sense are those satisfied by the diagrams $m=|\cup|$ and $u=\cap$, which in turn generate $T L_{n}$.

We recall now that the tensor powers of the fundamental representation of $P U_{2} \simeq \mathrm{SO}_{3}$ form a category which is isomorphic to $T L_{4}$. This shows that the irreducible representations of $A_{s}(4)$, with fusion rules and dimensions, are the same as irreducible representations of $\mathrm{SO}_{3}$. Moreover, the fusion rule statement must hold for any $n \geq 4$.

THEOREM 7.2. For any $n \geq 4$, the irreducible corepresentations of $A_{s}(n)$ satisfy the Clebsch-Gordan rules for irreducible representations of $\mathrm{SO}_{3}$.

In other words, the irreducible corepresentations are as follows.

1. They are given by $r_{0}=1, r_{1}=u-1, r_{2}=u^{\otimes 2}-3 u+1$ and so on.

2. They satisfy $r_{a} \otimes r_{b}=r_{|a-b|}+r_{|a-b|+1}+\ldots+r_{a+b-1}+r_{a+b}$.

These considerations have several extensions. We would like to mention here the following statement, which trivializes the whole thing: the quotients of $A_{s}(n)$ are in functorial correspondence with subalgebras of the $n$-th spin planar algebra. See [3]. 
8. The Weingarten formula. By general results of Woronowicz in [58], the Hopf algebra $A_{s}(n)$ has a unique unital bi-invariant state, called Haar integration, and denoted here as an integral:

$$
\int: A_{s}(n) \rightarrow \mathbb{C}
$$

The various integrals can be computed by using the representation theory diagrams found in previous section. The idea here, going back to Weingarten's paper [57], was developed in [16], [27], [28], [29], and was applied to quantum groups in [10], [11], [12].

Definition 8.1. Consider the set $N C(k)$ of non-crossing partitions of $\{1, \ldots, k\}$.

1. We plug multi-indices $i=\left(i_{1}, \ldots, i_{k}\right)$ into partitions $p \in N C(k)$, and we set $\delta_{p i}=1$ if all blocks of $p$ contain equal indices of $i$, and $\delta_{p i}=0$ if not.

2. The Gram matrix of partitions (of index $n \geq 4$ ) is given by $G_{k n}(p, q)=n^{|p \vee q|}$, where $\vee$ is the set-theoretic sup, and $|$.$| is the number of blocks.$

3. The Weingarten matrix $W_{k n}$ is the inverse of $G_{k n}$.

The non-crossing partitions are in correspondence with Temperley-Lieb diagrams having no upper points: these can be indeed obtained by fattening the partitions.

Now by using the Temperley-Lieb action described in the previous section, we see that the elements of $N C(k)$ create a basis of fixed vectors of $u^{\otimes k}$. The Gram matrix of this basis is nothing but $G_{k n}$, as shown by the following computation:

$$
\langle p, q\rangle=\sum_{i} \delta_{p i} \delta_{q i}=\sum_{i} \delta_{p \vee q, i}=n^{|p \vee q|} .
$$

Observe also that $G_{k n}$ is by definition a kind of version of Di Francesco's meander matrix in [33]. With these notations, we have the following result.

THEOREM 8.1. The Haar functional of $A_{s}(n)$ is given by

$$
\int u_{i_{1} j_{1}} \ldots u_{i_{k} j_{k}}=\sum_{p q} \delta_{p i} \delta_{q j} W_{k n}(p, q)
$$

where the sum is over all pairs of diagrams $p, q \in N C(k)$.

The proof is based on the following fact: the numbers on the left are the matrix coefficients of the orthogonal projection onto the space of fixed points of $u^{\otimes k}$.

As a first consequence, we have the following moment formula:

$$
\int\left(u_{11}+\ldots+u_{s s}\right)^{k}=\operatorname{Tr}\left(G_{k n}^{-1} G_{k s}\right) .
$$

The free Poisson law of parameter $t \in(0,1]$ is the following probability measure:

$$
\pi_{t}=(1-t) \delta_{0}+\frac{1}{2 \pi x} \sqrt{4 t-(x-1-t)^{2}} d x .
$$

This measure is also called Marchenko-Pastur law. The terminology here comes from the fact that $\pi_{t}$ is the free analogue of the Poisson law of parameter $t$. See [53], [54].

THEOREM 8.2. With $n \rightarrow \infty$ the law of $u_{11}+\ldots+u_{s s}$ with $s=[$ tn $]$ converges to $\pi_{t}$. 
This follows from the moment formula, by using the fact that with $n \rightarrow \infty$, both the Gram and Weingarten matrices are concentrated on the diagonal. The trace to be computed reduces to a sum of powers of $t$, known to give the $k$-th moment of $\pi_{t}$.

In the classical case a similar result is available, in terms of Poisson laws. As a conclusion, $C\left(S_{n}\right) \rightarrow A_{s}(n)$ transforms asymptotic independence into freeness. See [11].

9. The Pauli quantum group. The central object of the theory is the algebra $A_{s}(4)$. In this section we present an explicit matrix model for this algebra, coming from the Pauli matrices:

$$
c_{1}=\left(\begin{array}{ll}
1 & 0 \\
0 & 1
\end{array}\right) \quad c_{2}=\left(\begin{array}{cc}
i & 0 \\
0 & -i
\end{array}\right) \quad c_{3}=\left(\begin{array}{cc}
0 & 1 \\
-1 & 0
\end{array}\right) \quad c_{4}=\left(\begin{array}{ll}
0 & i \\
i & 0
\end{array}\right) .
$$

These matrices multiply according to the formulae for quaternions:

$$
\begin{aligned}
& c_{2}^{2}=c_{3}^{2}=c_{4}^{2}=-1, \\
& c_{2} c_{3}=-c_{3} c_{2}=c_{4}, \\
& c_{3} c_{4}=-c_{4} c_{3}=c_{2}, \\
& c_{4} c_{2}=-c_{2} c_{4}=c_{3} .
\end{aligned}
$$

The Pauli matrices form an orthonormal basis of $M_{2}(\mathbb{C})$, and the same is true if we multiply them to the left or to the right by an element of $S U_{2}$. This shows that for any $x \in S U_{2}$, the elements $\xi_{i j}=c_{i} x c_{j}$ form a magic basis of $M_{2}(\mathbb{C})$, in the sense that the corresponding orthogonal projections $P_{i j}$ form a magic unitary over $M_{4}(\mathbb{C})$.

Definition 9.1. The Pauli representation is the map

$$
\pi: A_{s}(4) \rightarrow C\left(S U_{2}, M_{4}(\mathbb{C})\right)
$$

given by $\pi\left(u_{i j}\right)=\left(x \mapsto\right.$ rank one projection on $\left.c_{i} x c_{j}\right)$.

This representation is introduced in [13]. In [12] we use integration techniques for proving that $\pi$ is faithful. The idea is to check commutativity of the following diagram:

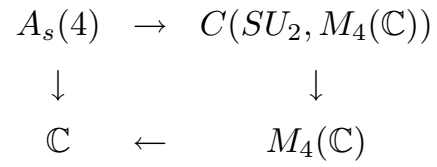

A key problem is to work out the integral geometric analogy between $C\left(S_{4}\right)$ and $A_{s}(4)$, at level of laws of averages of diagonal coordinates $u_{i i}$. For $C\left(S_{4}\right)$ we have:

$$
\operatorname{law}\left(t_{1} u_{11}+\ldots+t_{4} u_{44}\right)=\frac{1}{24}\left(9 \delta_{0}+\delta_{1}+2 \sum_{i} \delta_{t_{i}}+\sum_{i \neq j} \delta_{t_{i}+t_{j}}\right) .
$$

For the algebra $A_{s}(4)$ we can use the Pauli representation, which makes integration problems correspond to computations on the real sphere $S^{3}$.

THEOREM 9.1. For $s=1,2,4$ we have the formula

$$
\operatorname{law}\left(s^{-1}\left(u_{11}+\ldots+u_{s s}\right)\right)=\left(1-\frac{s}{4}\right) \delta_{0}+\frac{s}{4} \mu_{s}
$$

where $\mu_{1}, \mu_{2}, \mu_{4}$ are a Dirac mass, a Lebesgue measure, and a free Poisson law. 
This result doesn't quite clarify the relation with $C\left(S_{4}\right)$. In fact, what is missing is the $s=3$ law. This is the law of the following matrix, depending on $(a, b, c, d) \in S^{3}$ :

$$
M_{3}=\frac{1}{3}\left(\begin{array}{cccc}
3 a^{2} & -a b & -a c & -a d \\
-a b & 3 b^{2} & -b c & -b d \\
-a c & -b c & 3 c^{2} & -c d \\
-a d & -b d & -c d & 3 d^{2}
\end{array}\right) .
$$

We don't know how to compute this measure. The problem is explained in [12].

10. The hyperoctahedral quantum group. In this section we present a few known facts, along with some recent work [8]. The quantum symmetry algebra of a finite graph $X$ is defined as follows.

1. In case $X$ has no edges, we set $A(X)=A_{s}(n)$. The fact that this is indeed a quantum symmetry algebra follows from considerations in previous sections.

2. In the general case, we set $A(X)=A_{s}(n) / R$, where $R$ are the relations coming from $d u=u d$, where $d \in M_{n}(0,1)$ is the adjacency matrix of $X$.

The quotients of $A(X)$ are called Hopf algebras coacting on $X$. See [2], [4], [18].

Let us go back to the magic unitary matrix $u_{p q}$ in section 6 . This matrix commutes with the adjacency matrix of the square. Moreover, by choosing the projections $p, q$ to be free, the algebra $\langle p, q\rangle$ they generate is isomorphic to the group algebra of $Z_{2} * Z_{2}=D_{\infty}$, and we get the following result.

THEOREM 10.1. The dual of $D_{\infty}$ acts on the square.

In other words, for the square we have an arrow $A(X) \rightarrow C^{*}\left(D_{\infty}\right)$. The algebra $A(X)$ can be actually computed explicitly, and is isomorphic to $C\left(\mathrm{O}_{2}^{-1}\right)$. See [20].

This result has the following generalization. Consider the Hopf algebra $C\left(O_{n}^{-1}\right)$, which is the quotient of $A_{o}(n)$ by the skew-commutation relations for $G L_{n}^{-1}$, namely:

1. $u_{i j} u_{i k}=-u_{i k} u_{i j}, u_{j i} u_{k i}=-u_{k i} u_{j i}$, for $i \neq j$,

2. $u_{i j} u_{k l}=u_{k l} u_{i j}$ for $i \neq k, j \neq l$.

These relations define a Hopf ideal, so we have indeed a Hopf algebra.

THEOREM 10.2. $C\left(O_{n}^{-1}\right)$ is the quantum symmetry algebra of the hypercube in $\mathbb{R}^{n}$.

This leads to the quite surprising conclusion that $O_{n}^{-1}$ is a quantum analogue of the hyperoctahedral group $H_{n}$. On the other hand, $O_{n}^{-1}$ cannot be a free version of $H_{n}$, say because the fusion semi-ring depends on $n$, which avoids probabilistic freeness.

In order to solve this problem, the idea is as follows. The group $H_{n}$ appears also as symmetry group of the space formed by the $[-1,1]$ segments on each coordinate axis. In other words, $H_{n}$ is the symmetry group of $I_{n}$, the graph formed by $n$ segments.

Definition 10.1. $A_{h}(n)$ is the quantum symmetry algebra of $I_{n}$.

This algebra is the quotient of $A_{s}(2 n)$ by the relations coming from commutation of $u$ with the adjacency matrix of $I_{n}$. Now writing down the commutation relations leads 
to the conclusion that $u$ must be a magic unitary of the following special form:

$$
u=\left(\begin{array}{ll}
a & b \\
b & a
\end{array}\right) .
$$

We call such a matrix a $2 n \times 2 n$ sudoku unitary. With this notion in hand, $A_{h}(n)$ is the universal algebra generated by entries of a $2 n \times 2 n$ sudoku unitary.

As a first consequence, we have the following commutative diagram:

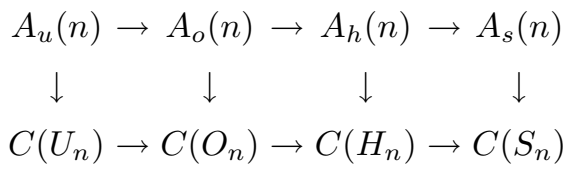

A Tannakian translation gives the representation theory of $A_{h}(n)$ : the relevant algebra is the Fuss-Catalan algebra of Bisch and Jones [22], and the Weingarten machinery leads to a free analogue of the Bessel function combinatorics for $H_{n}$.

11. Free wreath products. The simplest example of a wreath product is the hyperoctahedral group $H_{n}$. Consider the graph $I_{n}$ formed by $n$ segments, and for each segment $I^{i}$ consider the element $\tau_{i} \in H_{n}$ which returns $I^{i}$, and keeps the other segments fixed. The elements $\tau_{1} \ldots \tau_{n}$ have order two and commute with each other, so they generate a product $L_{n}=Z_{2} \times \ldots \times Z_{2}$. Now the symmetric group $S_{n}$ acts as well on $I_{n}$ by permuting the segments, and it is routine to check that we have $H_{n}=L_{n} \rtimes S_{n}$. But this latter group is a wreath product.

THEOREM 11.1. We have $H_{n}=Z_{2}$ ? $S_{n}$.

At level of algebras of functions, this gives a formula of the following type, where $\times_{w}$ is some functional analytic implementation of the 2 operation:

$$
C\left(H_{n}\right)=C\left(Z_{2}\right) \times{ }_{w} C\left(S_{n}\right) .
$$

The operation $\times_{w}$ is not the good one for quantum groups. This is because the natural quantum formula involving it would imply $A_{h}(2)=C\left(H_{2}\right)$, which is wrong. The point is that in the quantum world, wreath products are replaced by free wreath products.

DeFinition 11.1. The free wreath product of $(A, u)$ and $(B, v)$ is given by

$$
A *_{w} B=\left(A^{* n} * B\right) /<\left[u_{i j}^{(a)}, v_{a b}\right]=0>
$$

where $n$ is the size of $v$, and has magic unitary matrix $w_{i a, j b}=u_{i j}^{(a)} v_{a b}$.

This notion, introduced in [20], is justified by formulae of the following type, where $G, A$ denote classical symmetry groups, respectively quantum symmetry algebras:

$$
G(X * Y)=G(X) \text { 乙 } G(Y), \quad A(X * Y)=A(X) *_{w} A(Y) .
$$

There are several such formulae, and the one we are interested in is:

$$
\begin{aligned}
& G(I \ldots I)=G(I) \quad \text { } \quad G(\circ \ldots \circ), \\
& A(I \ldots I)=A(I) *_{w} A(\circ \ldots \circ) .
\end{aligned}
$$


Here $I$ is a segment, $\circ$ is a point, and the dots mean $n$-fold disjoint union. The first formula is $H_{n}=Z_{2} 2 S_{n}$. As for the second formula, this is what we need.

TheOrem 11.2. We have $A_{h}(n)=C\left(Z_{2}\right) *{ }_{w} A_{s}(n)$.

Now getting back to general free wreath products, a first thing to be noticed is the following diagram, where maps on the left are defined by formulae on the right:

$$
\begin{aligned}
A^{* n} * B & \rightarrow A *_{w} B & \sum u_{i i}^{(a)} v_{a a} & \rightarrow \sum u_{i i}^{(a)} v_{a a} \\
\downarrow & \downarrow & \downarrow & \downarrow \\
A * B & \rightarrow A \otimes B & \sum u_{i i} v_{a a} & \rightarrow \sum u_{i i} \otimes v_{a a}
\end{aligned}
$$

The spectral measures of the north-east and south-west elements can be computed in several cases of interest, and turn out to be equal. The conjecture is that equality holds, under general assumptions. This is the same as saying that the spectral measure of $A *_{w} B$ appears as free multiplicative convolution of the spectral measures of $A, B$ :

$$
\mu\left(A *_{w} B\right)=\mu(A) \otimes \mu(B) .
$$

This is related to work in preparation of Bisch and Jones, see [4]. The whole thing would be a first step towards establishing an analogy with results of Sniady [45].

12. Quantum automorphisms of finite graphs. The free wreath product results in previous section are part of a classification project for finite graphs [2], [3], [4], [5]. This is in turn part of the Bisch-Jones classification project for planar algebras generated by a 2-box [23], [24]. Indeed, it follows from the general Tannaka-Galois duality in [3] that the algebras of form $A(X)$ with $X$ colored oriented graph are those corresponding to subalgebras of the spin planar algebra, generated by a 2-box.

The conclusion in [5] is the following table, containing all vertex-transitive graphs of order $n \leq 11$ modulo complementation, except for the Petersen graph.

\begin{tabular}{llll}
\hline Order & Graph & Classical group & Quantum group \\
\hline 2 & $K_{2}$ (simplex) & $Z_{2}$ & $Z_{2}$ \\
3 & $K_{3}$ & $S_{3}$ & $S_{3}$ \\
4 & $2 K_{2}$ (duplication) & $H_{2}$ & $H_{2}^{+}$(hyper. quant. group) \\
4 & $K_{4}$ & $S_{4}$ & $S_{4}^{+}$(symm. quant. group) \\
5 & $C_{5}$ (cycle) & $D_{5}$ & $D_{5}$ \\
5 & $K_{5}$ & $S_{5}$ & $S_{5}^{+}$ \\
6 & $C_{6}$ & $D_{6}$ & $D_{6}$ \\
6 & $2 K_{3}$ & $S_{3} \succ Z_{2}$ & $S_{3} 2_{*} Z_{2}$ (free wreath prod.) \\
6 & $3 K_{2}$ & $H_{3}$ & $H_{3}^{+}$ \\
6 & $K_{6}$ & $S_{6}$ & $S_{6}^{+}$ \\
7 & $C_{7}$ & $D_{7}$ & $D_{7}$ \\
7 & $K_{7}$ & $S_{7}$ & $S_{7}^{+}$ \\
\hline
\end{tabular}




\begin{tabular}{|c|c|c|c|}
\hline Order & Graph & Classical group & Quantum group \\
\hline 8 & $C_{8}, C_{8}^{+}$(cycle with diags.) & $D_{8}$ & $D_{8}$ \\
\hline 8 & $P\left(C_{4}\right)($ prism $)$ & $H_{3}$ & $S_{4}^{+} \times Z_{2}$ \\
\hline 8 & $2 K_{4}$ & $S_{4} \prec Z_{2}$ & $S_{4}^{+} \imath_{*} Z_{2}$ \\
\hline 8 & $2 C_{4}$ & $H_{2} \succ Z_{2}$ & $H_{2}^{+} \imath_{*} Z_{2}$ \\
\hline 8 & $4 K_{2}$ & $H_{4}$ & $H_{4}^{+}$ \\
\hline 8 & $K_{8}$ & $S_{8}$ & $S_{8}^{+}$ \\
\hline 9 & $C_{9}, C_{9}^{3}$ (cycle with chords) & $D_{9}$ & $D_{9}$ \\
\hline 9 & $K_{3} \times K_{3}$ (discrete torus) & $S_{3} \succ Z_{2}$ & $S_{3} \succ Z_{2}$ \\
\hline 9 & $3 K_{3}$ & $S_{3} \prec S_{3}$ & $S_{3} 2_{*} S_{3}$ \\
\hline 9 & $K_{9}$ & $S_{9}$ & $S_{9}^{+}$ \\
\hline 10 & $C_{10}, C_{10}^{2}, C_{10}^{+}, P\left(C_{5}\right)$ & $D_{10}$ & $D_{10}$ \\
\hline 10 & $P\left(K_{5}\right)$ & $S_{5} \times Z_{2}$ & $S_{5}^{+} \times Z_{2}$ \\
\hline 10 & $C_{10}^{4}$ & $Z_{2} \prec D_{5}$ & $Z_{2} \imath_{*} D_{5}$ \\
\hline 10 & $2 C_{5}$ & $D_{5} \succ Z_{2}$ & $D_{5} 2_{*} Z_{2}$ \\
\hline 10 & $2 K_{5}$ & $S_{5} \succ Z_{2}$ & $S_{5}^{+} \imath_{*} Z_{2}$ \\
\hline 10 & $5 K_{2}$ & $H_{5}$ & $H_{5}^{+}$ \\
\hline 10 & $K_{10}$ & $S_{10}$ & $S_{10}^{+}$ \\
\hline 11 & $C_{11}, C_{11}^{2}, C_{11}^{3}$ & $D_{11}$ & $D_{11}$ \\
\hline 11 & $K_{11}$ & $S_{11}$ & $S_{11}^{+}$ \\
\hline
\end{tabular}

13. Graphs having no quantum symmetry. The classification project for finite graphs is there for various reasons, one of them being to help in classification of certain subfactors and planar algebras, of integer index. In other words, the whole thing should be regarded as belonging to a specialized area of von Neumann algebras, and mathematical physics in general.

As explained to us by Jones, the end of the game would be to investigate some special graphs, such as the Clebsch graph, or the Higman-Sims graph.

We are quite far away from this kind of application. The big list in previous section consists of simplexes, cycles, and their products. That is, our main realization so far is to have reasonably strong results about product operations.

The next step would be to develop some new techniques, for graphs which do not decompose as products. The first such graph is the Petersen one, at $n=10$. As already mentioned, we have no results about it. But work here is in progress, and we hope to come up soon with an answer, along with a study for higher $n$, say between 12-15.

We would like to present now a first conceptual result emerging from our small $n$ study. This concerns graphs having no quantum symmetry.

Definition 13.1. A finite graph $X$ has no quantum symmetry if it satisfies one of the following equivalent conditions, where $d$ is its adjacency matrix.

1. The quantum symmetry algebra $A(X)$ is commutative.

2. We have $A(X)=C\left(G_{X}\right)$, where $G_{X}$ is the symmetry group of $X$.

3. For a magic unitary $u, d u=u d$ implies that $u_{i j}$ commute with each other. 
The problem of characterizing such graphs goes back to Wang's paper [56], with the results $A\left(C_{3}\right)=C\left(S_{3}\right)$ and $A\left(C_{4}\right) \neq C\left(H_{2}\right)$, showing that $C_{3}$ has no quantum symmetry, but $C_{4}$ does. A topological formulation of the problem is found by Curtin in [32].

There are several graphs in the above table which satisfy this condition: the cycles $C_{n}$ with $n \neq 4$, a number of prisms and of cycles with chords, and the discrete torus $K_{3} \times K_{3}$. Moreover, we have found some more graphs by working on the subject, and this led us to take a detailed look at the case of circulant graphs.

A graph $X$ having $n$ vertices is called circulant if its automorphism group contains copy of $Z_{n}$. This is the same as saying that vertices of $X$ are elements of $Z_{n}$, and that $i \sim j$ (connection by an edge) implies $i+k \sim j+k$ for any $k$.

Associated to a circulant graph are the following algebraic invariants.

1. The set $S \subset Z_{n}$ is given by $i \sim j \Longleftrightarrow j-i \in S$.

2. The group $E \subset Z_{n}^{*}$ consists of elements $a$ such that $a S=S$.

3. The order of $E$ is denoted $k$, and is called type of $X$.

With these notations, we have the following result:

THEOREM 13.1. A type $k$ circulant graph having $p \gg k$ vertices, with p prime, has no quantum symmetry.

This result is proved in [7], with the lower bound $p>6^{\varphi(k)}$, where $\varphi$ is the Euler function. Most of the proof there doesn't really use the fact that $p$ is prime, and we hope to come up soon with more general results in this sense.

The whole subject is somehow opposite to the freeness considerations in previous sections: "no quantum symmetry" means "too many constrains, hence independence".

14. Hadamard matrices. A complex Hadamard matrix is a matrix $h \in M_{n}(\mathbb{C})$ having the following properties: entries are on the unit circle, and rows are mutually orthogonal.

The rows of $h$, denoted $h_{1}, \ldots, h_{n}$, can be regarded as elements of the algebra $\mathbb{C}^{n}$. Since each $h_{i}$ is formed by complex numbers of modulus 1 , this element is invertible. We can therefore consider the elements $\xi_{i j}=h_{j} / h_{i}$, and we have:

$$
\left\langle\xi_{i j}, \xi_{i k}\right\rangle=\left\langle h_{j} / h_{i}, h_{k} / h_{i}\right\rangle=n\left\langle h_{j}, h_{k}\right\rangle=n \cdot \delta_{j k} .
$$

A similar computation works for columns, so $\xi$ is a magic basis of $\mathbb{C}^{n}$, in the sense that the corresponding orthogonal projections $P\left(\xi_{i j}\right)$ form a magic unitary matrix.

Definition 14.1. Let $h \in M_{n}(\mathbb{C})$ be an Hadamard matrix.

1. $\xi$ is the magic basis of $\mathbb{C}^{n}$ given by $\xi_{i j}=h_{j} / h_{i}$.

2. $P$ is the magic unitary over $M_{n}(\mathbb{C})$ given by $P_{i j}=P\left(\xi_{i j}\right)$.

3. $\pi$ is the representation of $A_{s}(n)$ given by $\pi\left(u_{i j}\right)=P_{i j}$.

4. $A$ is the quantum permutation algebra associated to $\pi$.

In other words, we say that the representation $\pi: A_{s}(n) \rightarrow M_{n}(\mathbb{C})$ comes from a representation $\nu: G_{n} \rightarrow U_{n}$ of the dual of the $n$-th quantum permutation group, then we consider the quantum group $G=\nu\left(G_{n}\right)$, and the algebra $A=C^{*}(G)$. See [14].

THEOREM 14.1. The construction $h \rightarrow A$ has the following properties. 
1. The Fourier matrix $h_{i j}=w^{i j}$ with $w=e^{2 \pi i / n}$ gives $A=C\left(Z_{n}\right)$.

2. For a tensor product $h=h^{\prime} \otimes h^{\prime \prime}$ we have $A=A^{\prime} \otimes A^{\prime \prime}$.

3. A is commutative if and only if $h$ is a tensor product of Fourier matrices.

For $n=1,2,3,5$ any Hadamard matrix is equivalent to the Fourier one. At $n=4$ we have the following example, depending on $q$ on the unit circle:

$$
h_{q}=\left(\begin{array}{cccc}
1 & 1 & 1 & 1 \\
1 & q & -1 & -q \\
1 & -1 & 1 & -1 \\
1 & -q & -1 & q
\end{array}\right) .
$$

These are, up to equivalence, all $4 \times 4$ Hadamard matrices. As an example, the Fourier matrix corresponds to the value $q= \pm i$. See Haagerup [39].

THEOREM 14.2. Let $n$ be the order of $q^{2}$, and for $n<\infty$ write $n=2^{s} m$, with $m$ odd. The matrix $h_{q}$ produces the algebra $C^{*}(G)$, where $G$ is as follows.

1. For $s=0$ we have $G=Z_{2 n} \rtimes Z_{2}$.

2. For $s=1$ we have $G=Z_{n / 2} \rtimes Z_{4}$.

3. For $s \geq 2$ we have $G=Z_{n} \rtimes Z_{4}$.

4. For $n=\infty$ we have $G=Z \rtimes Z_{2}$.

This result provides the first example of a deformation situation for quantum permutation groups. Observe that the parameter space is the unit circle, with roots of unity highlighted. We should mention that this space, while being fundamental in most theories emerging from Drinfeld's original work [35], is quite new in the compact quantum group area, where the deformation parameter is traditionally real. See [14].

There are many difficult problems regarding Hadamard matrices, and we don't know yet if quantum permutation groups can help. See Jones [41].

15. Cocycle twists of the symmetric group. The examples of quantum permutation groups discussed so far in this paper are either classical or infinite-dimensional. A natural question is whether there exist non-classical finite quantum permutation groups. The construction of such objects was done in [17], using the 2-cocycle twisting procedure.

The idea of twisting, originally due to Drinfeld [36], and developed by Doi [34] in the dual framework, is the following one. Starting with a Hopf algebra $A$, we consider linear maps $\sigma: A \otimes A \rightarrow \mathbb{C}$ satisfying certain conditions and called (Hopf) 2-cocycles. We then deform the product of $A$ by $\sigma$ to get a new Hopf algebra $A^{\sigma}$, called a twist of $A$, and having the same tensor category of corepresentations as $A$. See Schauenburg [44].

The theory of twisting is developed at different levels of generality and studied in numerous papers that we shall not list here. Amongst these, the paper of Enock and Vainerman [38] was influential: they realized that 2-cocycles could be easily constructed from abelian subgroups, over which Hopf 2-cocycles correspond to ordinary group 2cocycles. This idea was used in [17] to construct twists of $C\left(S_{2 n}\right)$ induced by the abelian subgroup $Z_{2}^{n}$, leading to the following construction. 
Let $i \in\{1, \ldots, 2 n\}$. For $i$ even we put $i^{\prime}=i-1$ and $i^{*}=i / 2$. For $i$ odd we put $i^{\prime}=i+1$ and $i^{*}=i^{\prime} / 2$. We consider a matrix $p=\left(p_{i j}\right) \in M_{n}(\mathbb{C})$ with $p_{i i}=1$ and $p_{i j}=p_{j i}= \pm 1$ for all $i$ and $j$.

Definition 15.1. The Hopf algebra $C_{p}\left(S_{2 n}\right)$ is defined to be the quotient of $A_{s}(2 n)$ by the following relations:

$$
\begin{aligned}
& \left(3+p_{i^{*} j^{*}}\right) u_{k j} u_{l i}+\left(1-p_{i^{*} j^{*}}\right) u_{k j} u_{l i^{\prime}}+\left(1-p_{i^{*} j^{*}}\right) u_{k j^{\prime}} u_{l i}+\left(p_{i^{*} j^{*}}-1\right) u_{k j^{\prime}} u_{l i^{\prime}} \\
& =\left(3+p_{l^{*} k^{*}}\right) u_{l i} u_{k j}+\left(1-p_{l^{*} k^{*}}\right) u_{l^{\prime} i} u_{k j}+\left(1-p_{l^{*} k^{*}}\right) u_{l i} u_{k^{\prime} j}+\left(p_{l^{*} k^{*}}-1\right) u_{l^{\prime} i} u_{k^{\prime} j} .
\end{aligned}
$$

The conceptual meaning of these relations is that they are FRT-relations [43] associated with a Yang-Baxter operator $\mathbb{C}^{2 n} \otimes \mathbb{C}^{2 n} \rightarrow \mathbb{C}^{2 n} \otimes \mathbb{C}^{2 n}$ attached to $p$.

One shows that $C_{p}\left(S_{2 n}\right)$ is a twist of $C\left(S_{2 n}\right)$ and here is the conclusion in [17].

THEOREM 15.1. There exist at least $n$ finite quantum permutation groups acting on $2 n$ points, and having the same tensor category of representations as $S_{2 n}$.

It is also possible to construct twists of $C\left(S_{2 n+1}\right)$ in the same manner. More general twistings of $S_{n}$ are constructed in [19], using arbitrary roots of unity.

We have the following natural questions.

1. Does there exist a finite graph having a non-classical finite quantum symmetry group, for example one of the above ones constructed by twisting?

2. Consider a finite quantum group $G$ obtained as a twisting of $S_{n}$. Is it true that $G$ is a quantum permutation group?

The twisting construction is also available for any finite group. This leads to some surprises: although the alternating group $A_{5}$ does not act faithfully on 4 points, it has a finite quantum analogue that does. This plays an important role in the classification of the quantum groups acting on 4 points in the next section.

Finally we should mention that the twisting procedure is also a useful tool to understand the infinite-dimensional situation: for example the Hopf algebras $C\left(O_{n}^{-1}\right)$ are twists of $C\left(O_{n}\right)$. As explained in the next section, in the case $n=4$, twisting techniques essentially enable us to classify the quantum groups acting on 4 points.

16. Quantum groups acting on 4 points. A natural problem in the area of quantum permutation groups is the classification problem, at least for small $n$. This is the same as the classification of Hopf algebra quotients of $A_{s}(n)$. In the dual language, we have to classify the quantum subgroups of $S_{n}^{+}$, the compact quantum group dual to $A_{s}(n)$, defined by $A_{s}(n)=C\left(S_{n}^{+}\right)$.

At $n=4$ we have the following result [6].

THEOREM 16.1. The compact quantum subgroups of $S_{4}^{+}$are as follows:

1. $S_{4}^{+} \simeq S O_{3}^{-1}$.

2. The quantum orthogonal group $\mathrm{O}_{2}^{-1}$.

3. The quantum group $\widehat{D}_{\infty}$, the quantum dual of the infinite dihedral group.

4. The symmetric group $S_{4}$ and its subgroups.

5. The quantum group $S_{4}^{\tau}$, the unique non-trivial twist of $S_{4}$. 
6. The quantum group $A_{5}^{\tau}$, the unique non-trivial twist of the alternating group $A_{5}$.

7. The quantum group $D_{n}^{\tau}, n$ even and $n \geq 6$, the unique non-trivial twist of the dihedral group of order $2 n$.

8. The quantum group $D C_{n}^{\tau}$ of order $4 n, n \geq 2$, a pseudo-twist of the dicyclic group of order $4 n$.

9. The quantum group $\widehat{D}_{n}, n \geq 3$, the quantum dual of the dihedral group of order $2 n$.

The first step in the proof is to show that $A_{s}(4)$ is in fact isomorphic with $C\left(S O_{3}^{-1}\right)$, the latter being the quotient of $C\left(S U_{3}^{-1}\right)$ by the relations making the fundamental matrix orthogonal. Then one shows that $C\left(\mathrm{SO}_{3}^{-1}\right)$ is a twist of $C\left(\mathrm{SO}_{3}\right)$ (recall that, in contrast, $C\left(S U_{2}^{-1}\right)$ is not a twist of $\left.C\left(S U_{2}\right)\right)$. Then one uses twisting techniques to show that the quantum subgroups diagonally containing the Klein subgroup of $\mathrm{SO}_{3}^{-1}$ correspond to twists of subgroups of $\mathrm{SO}_{3}$ containing the diagonal subgroup. The remaining cases are examined by using case-by-case arguments.

The existence and uniqueness of various quantum groups in the theorem follow from work of several authors, including Davydov, Etingof and Gelaki, Kac and Paljutkin, Masuoka, Nikshych, Vainerman.

We note that all the quantum groups occurring in the theorem were already known, and that the classification has lots of similarities with the one for the compact subgroups of $\mathrm{SO}_{3}$, which is explained by the twisting result.

A direct consequence of the classification theorem is the following result.

THEOREM 16.2. The classical symmetric group $S_{4}$ is maximal as a compact quantum subgroup of the quantum permutation group $S_{4}^{+}$.

We conjecture that for any $n$, the classical symmetric group $S_{n}$ is maximal in the quantum permutation group $S_{n}^{+}$.

The next step is to continue the classification for the next values of $n$, say $n=5,6,7$. At this stage we are very far from having complete results, or even from having a strategy. We expect that several additional technical difficulties will arise. The first one is the non-amenability of the discrete quantum group dual to $S_{n}^{+}$if $n \geq 5$, shown in [1].

As a last remark, the results presented here are in connection with the various $n=4$ results from previous sections. A first problem here would be to find explicit matrix models for all quantum groups in the above theorem. Another problem is to understand the relation with integration results, say via a systematic study of twisted integration.

17. Conclusion. In this paper we have presented several known facts about quantum permutation groups, most of them being published, or available at arxiv.org. The theory is quite recent: it originates from Wang's 1998 paper [56], and was basically developed in the last few years.

The meaning of these investigations might remain quite unclear. This is indeed the case: the whole subject, with all its possible interpretations, belongs to area of mathematical physics, where everything is by definition quite unclear. 
The problem is that the theory is not mature enough for a serious comparison with results in traditional theoretical physics. It is most likely that many years will pass before reaching to the correct technical level.

It is probably instructive here to recall the story of noncommutative geometry, which is illustrating for the difficulty of applying mathematical ideas. The theory was initiated by Connes in the early eighties, with ideas coming from foliations, groupoids, and the Atiyah-Singer theorem. The high energy physics motivation was revealed 10 years later, in the Connes-Lott paper [31]. This was still quite away from reasonable numeric results, and the $170 \mathrm{GeV}$ prediction for the mass of the Higgs boson was obtained 15 more years later, in the Chamseddine-Connes-Marcolli paper [26].

Back to quantum permutation groups, what we can say for the moment is that these encode, via a very simple formalism, a few recent results.

1. Relation with Jones theory. The idea here is to develop a double approach to the problem, in terms of subfactors and planar algebras, by using tools from functional analysis and low-dimensional topology. The first thing to be said is that the main problems concern the case of non-integer index, and that our study is quite far away from that (see [9]). However, in the simplest case, namely when the index is integer, quantum groups turn to appear, and we have reasons to believe that quantum permutation groups have to be investigated first. Among others, the results presented in this paper are in tune with the recent work of Bisch and Jones, in the case where the index is integer or generic.

2. Relation with Voiculescu theory. The idea here is to develop a systematic approach to freeness. Among several aspects of the theory we have Speicher's approach, in the spirit of Rota's treatment of classical probability [46], and Biane's representation theory approach [16]. Our results make appear a number of key notions of the theory, in the combinatorial framework. It is our hope that some further random matrix investigations will lead as well to analytic results.

3. Among other results, we have the pleasing appearance of the Pauli matrices in connection with the central object of the theory, namely the algebra $A_{s}(4)$. Another important fact is the appearance of Di Francesco's meander determinants in the context of integration problems. However, regarding both subjects, there is still a lot of work to be done before understanding why these objects appear as they do, in the context of quantum permutation groups.

On the mathematical side, there are a few directions to explore as well. A number of techniques coming from discrete groups are developed by Vaes, Vergnioux and their collaborators in [15], [21], [47], [48], [49], [50], [51], [52], for various types of discrete quantum groups. Some of these techniques are expected to apply to duals of quantum permutation groups, as a complement to the above-mentioned considerations.

\section{References}

[1] T. Banica, Symmetries of a generic coaction, Math. Ann. 314 (1999), 763-780.

[2] T. Banica, Quantum automorphism groups of small metric spaces, Pacific J. Math. 219 (2005), 27-51. 
[3] T. Banica, Quantum automorphism groups of homogeneous graphs, J. Funct. Anal. 224 (2005), 243-280.

[4] T. Banica and J. Bichon, Free product formulae for quantum permutation groups, J. Inst. Math. Jussieu, to appear.

[5] T. Banica and J. Bichon, Quantum automorphism groups of vertex-transitive graphs of order $\leq 11$, J. Algebraic Combin., to appear.

[6] T. Banica and J. Bichon, Quantum groups acting on 4 points, math.QA/0703118.

[7] T. Banica, J. Bichon and G. Chenevier, Graphs having no quantum symmetry, Ann. Inst. Fourier, to appear.

[8] T. Banica, J. Bichon and B. Collins, The hyperoctahedral quantum group, preprint math.RT/0701859.

[9] T. Banica and D. Bisch, Spectral measures of small index principal graphs, Comm. Math. Phys. 269 (2007), 259-281.

[10] T. Banica and B. Collins, Integration over compact quantum groups, Publ. Res. Inst. Math. Sci., to appear.

[11] T. Banica and B. Collins, Integration over quantum permutation groups, J. Funct. Anal. 242 (2007), 641-657.

[12] T. Banica and B. Collins, Integration over the Pauli quantum group, math.QA/0610041.

[13] T. Banica and S. Moroianu, On the structure of quantum permutation groups, Proc. Amer. Math. Soc. 135 (2007), 21-29.

[14] T. Banica and R. Nicoara, Quantum groups and Hadamard matrices, Panamer. Math. J. 17 (2007), 1-24.

[15] T. Banica and R. Vergnioux, Growth estimates for discrete quantum groups, preprint math.OA/0611232.

[16] P. Biane, Representations of symmetric groups and free probability, Adv. Math. 138 (1998), 126-181.

[17] J. Bichon, Quelques nouvelles déformations du groupe symétrique, C. R. Acad. Sci. Paris 330 (2000), 761-764.

[18] J. Bichon, Quantum automorphism groups of finite graphs, Proc. Amer. Math. Soc. 131 (2003), 665-673.

[19] J. Bichon, Hopf-Galois systems, J. Algebra 264 (2003), 565-581.

[20] J. Bichon, Free wreath product by the quantum permutation group, Alg. Rep. Theory 7 (2004), 343-362.

[21] J. Bichon, A. De Rijdt and S. Vaes, Ergodic coactions with large multiplicity and monoidal equivalence of quantum groups, Comm. Math. Phys. 262 (2006), 703-728.

[22] D. Bisch and V. F. R. Jones, Algebras associated to intermediate subfactors, Invent. Math. 128 (1997), 89-157.

[23] D. Bisch and V. F. R. Jones, Singly generated planar algebras of small dimension, Duke Math. J. 101 (2000), 41-75.

[24] D. Bisch and V. F. R. Jones, Singly generated planar algebras of small dimension II, Adv. Math. 175 (2003), 297-318.

[25] L. Brown, Ext of certain free product $\mathrm{C}^{*}$-algebras, J. Operator Theory 6 (1981), 135-141.

[26] A. H. Chamseddine, A. Connes and M. Marcolli, Gravity and the standard model with neutrino mixing, hep-th/0610241.

[27] B. Collins, Moments and cumulants of polynomial random variables on unitary groups, the Itzykson-Zuber integral, and free probability, Int. Math. Res. Not. 17 (2003), 953-982.

[28] B. Collins and P. Sniady, Integration with respect to the Haar measure on the unitary, orthogonal and symplectic group, Comm. Math. Phys. 264 (2006), 773-795. 
[29] B. Collins and P. Śniady, Representations of Lie groups and random matrices, preprint math.PR/0610285.

[30] A. Connes, Noncommutative Geometry, Academic Press, 1994.

[31] A. Connes and J. Lott, Particle models and noncommutative geometry, Nuclear Phys. B Proc. Suppl. 18 (1990), 29-47.

[32] B. Curtin, Some planar algebras related to graphs, Pacific J. Math. 209 (2003), 231-248.

[33] P. Di Francesco, Meander determinants, Comm. Math. Phys. 191 (1998), 543-583.

[34] Y. Doi, Braided bialgebras and quadratic algebras, Comm. Algebra 21 (1993), 1731-1749.

[35] V. Drinfeld, Quantum groups, Proc. ICM Berkeley, 1986, 798-820.

[36] V. Drinfeld, Quasi-Hopf algebras, Leningrad Math. J. 1 (1990), 1419-1457.

[37] M. Enock and J.-M. Schwartz, Kac Algebras and Duality of Locally Compact Groups, Springer, 1992.

[38] M. Enock and L. Vainerman, Deformation of a Kac algebra by an abelian subgroup, Comm. Math. Phys. 178 (1996), 571-596.

[39] U. Haagerup, Orthogonal maximal abelian $*$-subalgebras of the $n \times n$ matrices, in: Operator Algebras and Quantum Field Theory, International Press, 1997, 296-323.

[40] V. F. R. Jones, Index for subfactors, Invent. Math. 72 (1983), 1-25.

[41] V. F. R. Jones, Planar algebras I, math.QA/9909027.

[42] K. McClanahan, $C^{*}$-algebras generated by elements of a unitary matrix, J. Funct. Anal. 107 (1992), 439-457.

[43] N. Yu. Reshetikhin, L. A. Takhtajan and L. D. Faddeev, Quantization of Lie groups and Lie algebras, Leningrad Math. J. 1 (1990), 193-225.

[44] P. Schauenburg, Hopf bigalois extensions, Comm. Algebra 24 (1996), 3797-3825.

[45] P. Śniady, Gaussian fluctuations of representations of wreath products, Infin. Dimens. Anal. Quantum Probab. Relat. Top. 9 (2006), 529-546.

[46] R. Speicher, Multiplicative functions on the lattice of noncrossing partitions and free convolution, Math. Ann. 298 (1994), 611-628.

[47] S. Vaes, Strictly outer actions of groups and quantum groups, J. Reine Angew. Math. 578 (2005), 147-184.

[48] S. Vaes and N. Vander Vennet, Identification of the Poisson and Martin boundaries of orthogonal discrete quantum groups, math.OA/0605489.

[49] S. Vaes and R. Vergnioux, The boundary of universal discrete quantum groups, exactness and factoriality, math.OA/0509706.

[50] R. Vergnioux, K-amenability for amalgamated free products of amenable discrete quantum groups, J. Funct. Anal. 212 (2004), 206-221.

[51] R. Vergnioux, Orientation of quantum Cayley trees and applications, J. Reine Angew. Math. 580 (2005), 101-138.

[52] R. Vergnioux, The property of rapid decay for discrete quantum groups, J. Operator Theory, to appear.

[53] D. V. Voiculescu, Lectures on free probability theory, Lecture Notes in Math. 1738, Springer, 2000, 279-349.

[54] D. V. Voiculescu, K. J. Dykema and A. Nica, Free Random Variables, AMS, 1992.

[55] S. Wang, Free products of compact quantum groups, Comm. Math. Phys. 167 (1995), 671-692.

[56] S. Wang, Quantum symmetry groups of finite spaces, Comm. Math. Phys. 195 (1998), 195-211.

[57] D. Weingarten, Asymptotic behavior of group integrals in the limit of infinite rank, J. Math. Phys. 19 (1978), 999-1001. 
[58] S. L. Woronowicz, Compact matrix pseudogroups, Comm. Math. Phys. 111 (1987), 613665.

[59] S. L. Woronowicz, Tannaka-Krein duality for compact matrix pseudogroups. Twisted $S U(N)$ groups, Invent. Math. 93 (1988), 35-76. 